\title{
The electrochemical deposition of polyaniline at pure aluminium: electrochemical activity and corrosion protection properties
}

\author{
Kenneth G. Conroy, Carmel B. Breslin *,1 \\ Department of Chemistry, National University of Ireland Maynooth, Maynooth, Co. Kildare, Ireland
}

Received 19 August 2002; received in revised form 7 November 2002

\begin{abstract}
Polyaniline films were electrodeposited at pure aluminium from a tosylic acid solution containing aniline. These polymer films exhibited similar characteristics as pure polyaniline electrosynthesized at an inert platinum electrode, when removed from their respective substrates and dissolved in NMP. Both polymers had similar molecular weights and similar UV-visible absorption spectra. However, the aluminium substrate had a considerable effect on the electrochemical activity of the films. The polyaniline films deposited at aluminium appeared to lose electroactivity and the electrochemical impedance data were governed by the oxidized aluminium substrate. This is consistent with a galvanic interaction between the polymer and the aluminium substrate, giving rise to oxidation of the aluminium and reduction of the polymer. The polyaniline deposits appeared to offer only a slight increase in the corrosion resistance of aluminium. Surface potential measurements, using a scanning vibrating probe, showed that attack initiated underneath the polymer under anodic polarization conditions, indicating that chloride anions diffuse across the polymer to react at the underlying aluminium substrate.
\end{abstract}

(C) 2002 Elsevier Science Ltd. All rights reserved.

Keywords: Aluminium; Corrosion; Polyaniline; Galvanic interaction; Scanning vibrating probe

\section{Introduction}

In recent years there has been increased interest in conducting polymers and the application of these materials in various fields, such as biochemical capacitors [1], rechargeable batteries [2] and in corrosion protection [3-5]. Polyaniline, the most studied of the conducting polymers, is highly stable, is synthesized easily, is highly conducting and is readily converted between its various oxidation states. These oxidation states differ from each other by the number of quinoid rings, which range from zero to two in the elementary unit of four rings, with the other rings being benzenoid. The inter-conversion between the emeraldine base, with three benzenoid rings, and the leucoemeraldine base (LB), with four benzenoid rings, and the inter-conversion between the base and conducting salt can be seen in \footnotetext{
3815 .

* Corresponding author. Tel.: +353-1-708-3770; fax: +353-1-708-

E-mail address: cb.breslin@may.ie (C.B. Breslin).

${ }^{1}$ ISE member.
}

Fig. 1. In all cases, electroneutrality of the polymer is maintained by the presence of counter anions.

Conducting polymers, such as polyaniline, are electrosynthesized easily at inert electrodes such as gold and platinum, but it is much more difficult to generate these polymers at aluminium electrodes. This is connected with the thin, but highly stable protective oxide, $\mathrm{Al}_{2} \mathrm{O}_{3}$, which forms on aluminium, and acts as a barrier inhibiting electron transfer and the polymerization process. Furthermore, this oxide grows through an anodization process in the acidic solutions normally employed in the electrosynthesis of conducting polymers.

However, there have been reports on the successful deposition of polypyrrole at pure aluminium and aluminium alloys. In the first of these reports, by Husler and Beck [6], it was shown that polypyrrole could be deposited at a pure aluminium electrode from aqueous electrolytes of oxalic acid and pyrrole. At low pyrrole concentrations, growth of the $\mathrm{Al}_{2} \mathrm{O}_{3}$ in parallel with the electropolymerization process was found giving rise to patchy deposits of the polymer. However, with high 


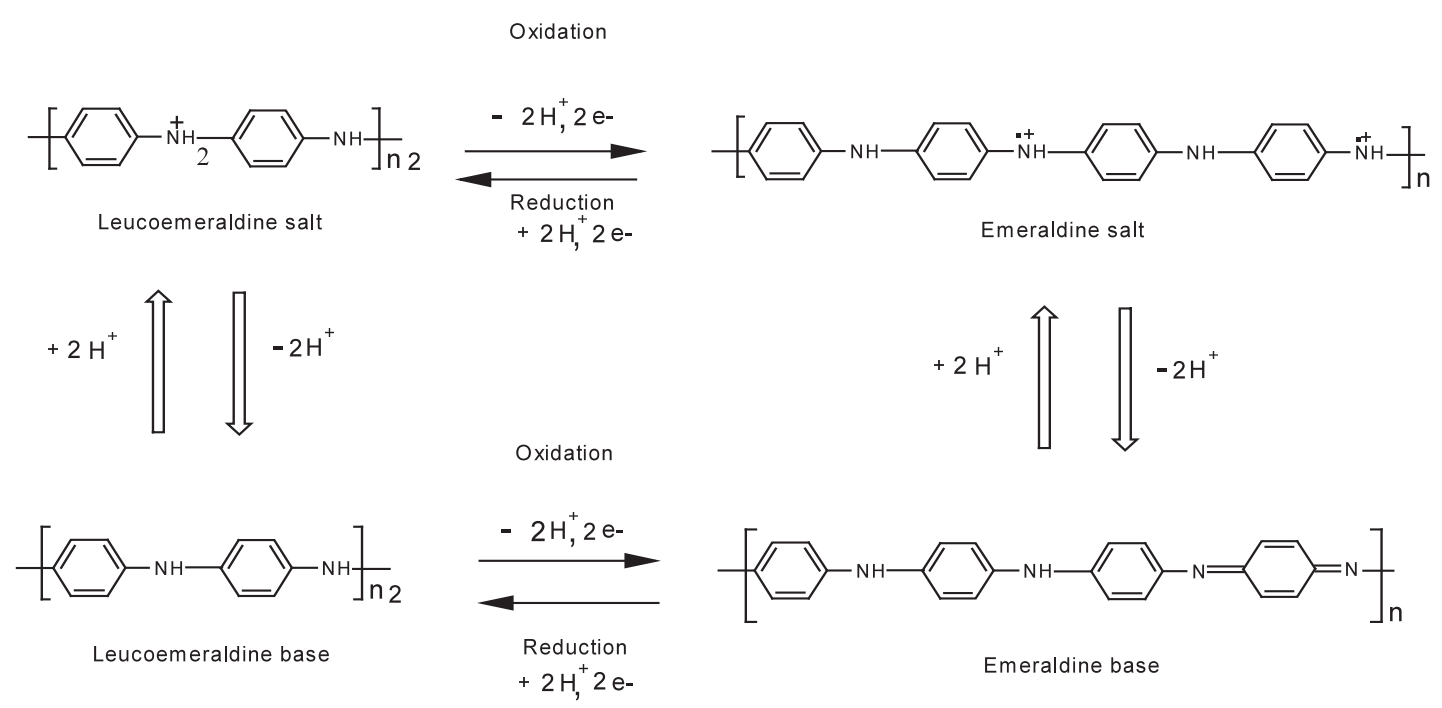

Fig. 1. Scheme showing the inter-conversion of polyaniline between the leucoemeraldine and emeraldine oxidation states and the inter-conversion between the salt and base states.

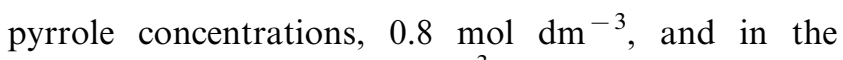

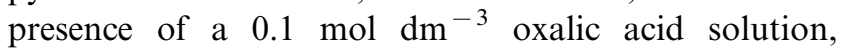
smooth, homogeneous and highly adherent films could be obtained. These authors also found that it was possible to deposit polypyrrole at aluminium from non-aqueous media such as acetonitrile and methanol [7].

Since these first findings, other reports have appeared in the literature on the deposition of conducting polypyrrole at aluminium, or aluminium alloys. For example, Tallman et al. [8] have electrodeposited polypyrrole at aluminium and an A12024-T3 alloy using 4,5dihydroxy-1,3-benzenedisulfonic acid di-sodium salt as a mediator to overcome kinetic limitations of the electron transfer at the insulating aluminium oxide surface. The mediator was found to reduce the deposition potential by nearly $500 \mathrm{mV}$ compared with that found in the absence of the mediator. Saidman and Bessone [9] have reported also the preparation of polypyrrole at aluminium from a nitric acid solution. They found that the coating was deposited initially at flaws in the passive aluminium oxide film. On studying the corrosion protection properties of these polypyrrole coatings, Saidman and Bessone found that localized corrosion of the underlying aluminium substrate occurred when the polymer-coated electrode was exposed to chloride anions. Naoi et al. [10] used various sulfonate-based electrolytes to prepare adherent polypyrrole at aluminium. They concluded that the aluminium was anodized in these solutions and the polypyrrole layers were generated with simultaneous formation of $\mathrm{Al}_{2} \mathrm{O}_{3}$.

There are, also, a number of reports in the literature in which polyaniline has been deposited at aluminium or aluminium alloys. However, these coatings have been prepared by casting the polyaniline from organic media onto the electrode. These works have centered mainly on the potential application of these cast coatings for corrosion protection. For example, Epstein et al. [11] have studied the corrosion protection properties of polyaniline in the emeraldine base form and self-doped sulfonated polyaniline cast-deposited at Al 3003 and A12024-T3 alloys. They found that these coatings were effective in reducing the corrosion rate when the coated electrodes were exposed to chloride environments. They also proposed that the polyaniline coatings facilitated the extraction of copper from the surface of the Al 2024T3 alloy, thereby reducing the galvanic couple between aluminium and copper and reducing the corrosion rate. Racicot et al. [12] have also reported the corrosion protection of aluminium alloys using a double-stranded polyaniline coating. They concluded that the conducting polymer was not a barrier coating, but that it converted the surface of the alloy to an oxidized state to form a passive layer that facilitated corrosion protection. Tallman et al. [13] have also shown that a polyaniline-epoxy two-coat system on Al 2024-T3 alloy gave good corrosion protection to the underlying alloy, while Shah et al. [14] found that the corrosion protection afforded by copolymer composites of polyaniline and polypyrrole depended on the electrochemical processing variables, such as the applied current density and the deposition period.

There have been much fewer reports on the direct preparation of polyaniline at an aluminium electrode. For example, Eftekhari $[15,16]$ has reported the preparation of enzyme-modified polyaniline coatings at an aluminium electrode from a $0.1 \mathrm{~mol} \mathrm{dm}^{-3}$ solution of aniline in a supporting sulfuric acid solution. However, this appears to be the only report on the direct electrochemical formation of polyaniline at an aluminium substrate. 
In this paper results are presented and discussed on the direct electropolymerization of aniline to produce adherent polyaniline films at pure aluminium from a tosylic acid solution. To the best of our knowledge, there are no reports in the literature detailing direct deposition of polyaniline, or indeed polypyrrole, at aluminium from such a solution. The characterization of these films using, UV-visible spectroscopy, gel permeation chromatography and electrochemical techniques, is reported and results on the corrosion protection properties of these electrosynthesized coatings are presented and discussed.

\section{Experimental}

\subsection{Materials}

Test specimens were prepared from high purity aluminium (99.9999\%). These samples were provided in rod form. Suitable lengths of the rods were embedded in epoxy resin in a Teflon holder to give a surface area of $0.785 \mathrm{~cm}^{2}$. Electrical contact was achieved by attaching a wire with the aid of a conductive epoxy to the aluminium rod.

\subsection{Electropolymerization}

Prior to electropolymerization, the samples were polished to a smooth surface finish, using successively finer grades of $\mathrm{SiC}$ paper, washed with distilled water and dried under an air stream. The polyaniline films were grown on aluminium at a constant potential in a conventional three-electrode cell for a period of $30 \mathrm{~min}$ at room temperature and in aqueous solutions containing 0.134 or $0.174 \mathrm{~mol} \mathrm{dm}^{-3}$ aniline and $1.0 \mathrm{~mol} \mathrm{dm}^{-3}$ tosylic acid ( $\mathrm{pH} 0.94)$ which were previously stirred. An EG\&G Potentiostat, Model 263A was used to provide the constant potential. A saturated calomel electrode was used as the reference electrode and high density and high surface area graphite rods were used as the counter electrodes. All potentials quoted are with respect to the saturated calomel reference electrode.

\subsection{Polymer analyses and corrosion measurements}

Once formed, the polymer-coated electrodes were washed in distilled water and then subjected to a series of electrochemical tests. These consisted of potentiodynamic polarization, cyclic voltammetry and electrochemical impedance tests. The impedance measurements were carried out using a Solartron Frequency Response Analyzer (Model SI 1250) in conjunction with the electrochemical interface EI 1286. All impedance data were recorded under open-circuit conditions using a sinusoidal excitation voltage of $10 \mathrm{mV}$. These data were fit to an appropriate equivalent circuit using a nonlinear least squares fitting routine using both the real and imaginary components of the data.

The potentiodynamic polarization tests were carried out using an EG\&G Potentiostat, Model 263 or a Solartron EI 1287 potentiostat. The potentiodynamic scans were carried out after an initial delay of $2 \mathrm{~min}$ using a scan rate of $1.0 \mathrm{mV} \mathrm{s}^{-1}$. The scans were initiated at $-1.6 \mathrm{~V}$ (SCE) and terminated at the breakdown potential, or some higher potential. These tests were performed in aggressive chloride-containing solutions with the chloride concentrations maintained at $0.5 \mathrm{~mol}$ $\mathrm{dm}^{-3}$. The $\mathrm{pH}$ of these solutions was modified using either $\mathrm{HCl}$ or $\mathrm{NaOH}$. Cyclic voltammograms were recorded in the monomer-free tosylic acid solution using a scan rate of $50 \mathrm{mV} \mathrm{s}^{-1}$ and cycling between -200 and $+750 \mathrm{mV}$ (SCE).

A scanning vibrating probe, SVP100, (EG\&G) was used to map localized electrochemical activity at the polyaniline coating. A twin platinum probe was scanned across the surface in the $x$ and $y$ directions at a constant height of $0.1 \mathrm{~mm}$ from the surface. The tip vibration amplitude was set at $50 \mu \mathrm{m}$ point to point and the tip velocity at $330 \mu \mathrm{m} \mathrm{s}^{-1}$. As the objective in this analysis was to induce corrosion of the relatively passive pure aluminium substrates, a potential was applied to the electrodes in order to induce attack of the pure aluminium substrates. In this case, a $5 \times 10^{-3} \mathrm{~mol}$ $\mathrm{dm}^{-3} \mathrm{NaCl}$ solution with a relatively low conductivity was employed for all analyses.

UV analyses were carried on a Cary UV-Vis 50 spectrophotometer. Polymer samples were exposed to air for 10-15 min and then dissolved in $N$-methyl-2pyrrolidinon (NMP), filtered to remove any insoluble polymer and then analyzed. The acid-base properties of the deposited polyaniline were monitored using solution $\mathrm{pH}$ measurements with a calibrated Orion $720 \mathrm{pH}$ meter. The molecular weights of the polyaniline deposits were measured using gel permeation chromatography. The polyaniline was dissolved in HPLC-grade NMP and filtered to remove any insoluble products prior to analyses. Polystyrene samples, with molecular masses between 780 and $114200 \mathrm{amu}$, were used as standards. The chromatograms were recorded using a PLgel column, $5 \mu \mathrm{m}$ MiniMIXC, using a flow rate of $0.2 \mathrm{ml}$ $\min ^{-1}$ operating at $50{ }^{\circ} \mathrm{C}$. A refractive index detector was employed.

\section{Results}

\subsection{Electrochemical synthesis}

A representative current-time plot showing the electrochemical synthesis of polyaniline, at a constant potential of $1.25 \mathrm{~V}$ (SCE), at pure aluminium in a 
supporting $1.0 \mathrm{~mol} \mathrm{dm}^{-3}$ tosylic acid solution is shown in Fig. 2. Two plots are presented; one showing the oxidation of pure aluminium in the absence of the monomer and the other recorded in a $0.174 \mathrm{~mol} \mathrm{dm}^{-3}$ aniline-containing tosylic acid solution. The data recorded in the absence of the monomer are characterized by an initial rapid decrease in the anodic current followed by a slower decay to reach a near-steady state condition. The data are very different in the presence of the monomer. The current initially decreases in a manner similar to that observed in the absence of the monomer, however after a short period, which can be equated with an induction period, the anodic current increases to reach a near-constant value. This increase in the anodic current, after the elapse of the induction period, marks the onset of the electropolymerization process and the deposition of polyaniline at the aluminium surface.

The induction period evident from the data in Fig. 2, marking the delay period prior to the polymer formation, was found to depend on the applied potential and concentration of the monomer. This can be seen from the data presented in Fig. 3( $a$ and $b$ ). The data presented in Fig. 3(a) were recorded in a $0.174 \mathrm{~mol} \mathrm{dm}^{-3}$ aniline

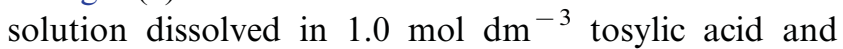
show the induction period plotted as a function of the applied potential. The data presented in Fig. 3(b) show the induction period as a function of the aniline concentration for electropolymerization at a constant applied potential of $1.25 \mathrm{~V}$ (SCE). It can be seen that high induction periods are measured at low aniline concentrations and at low applied potentials, conditions that favor the generation of a low concentration of radical cations, and accordingly a slow rate of electropolymerization.

It was also possible to form polyaniline films at aluminium in this medium using cyclic voltammetry. Representative cyclic voltammograms are shown in Fig. 4(a). These data were recorded between the potential

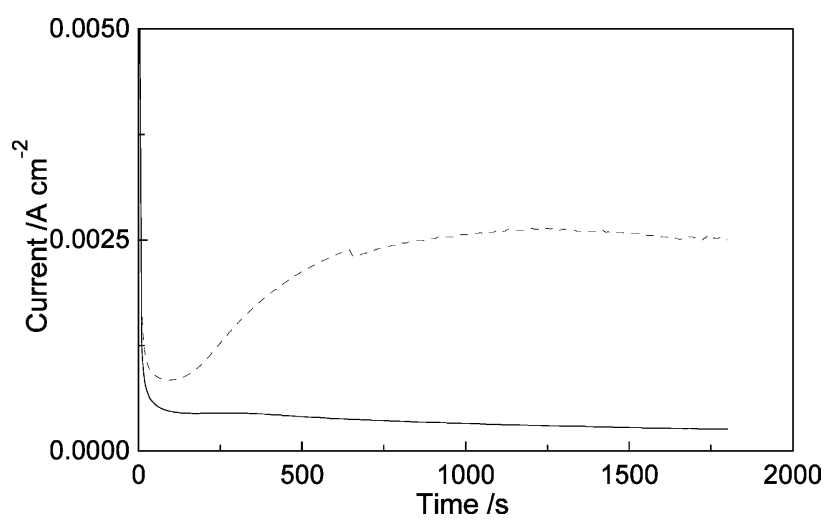

Fig. 2. Potentiostatic current-time plots recorded for aluminium at

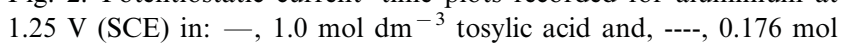
$\mathrm{dm}^{-3}$ aniline and $1.0 \mathrm{~mol} \mathrm{dm}{ }^{-3}$ tosylic acid.
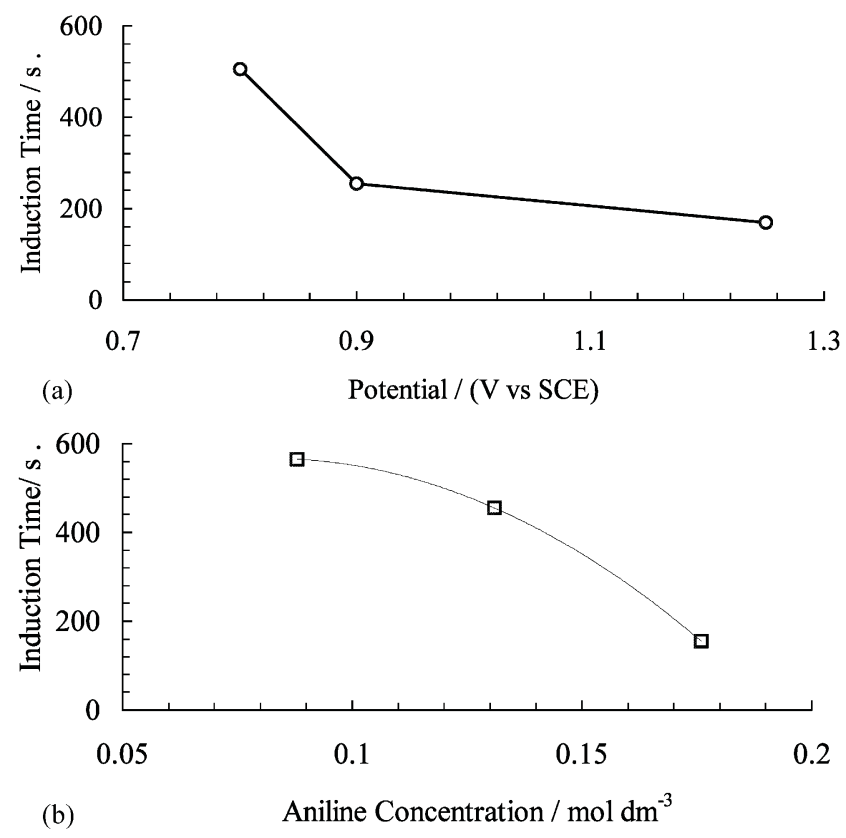

Fig. 3. (a) Plot showing the induction period plotted as a function of the electropolymerization potential for polymerization from a 0.176 mol dm ${ }^{-3}$ aniline and $1.0 \mathrm{~mol} \mathrm{dm}{ }^{-3}$ tosylic acid solution. (b) Plot showing the induction period plotted as a function of the aniline concentration for electropolymerization at $1.25 \mathrm{~V}$ (SCE) in a $1.0 \mathrm{~mol}$ $\mathrm{dm}^{-3}$ tosylic acid solution.

limits of -200 and $1250 \mathrm{mV}$ (SCE) and at a scan rate of $50 \mathrm{mV} \mathrm{s}^{-1}$. In this figure, three plots are shown, one depicting the second cycle, a second plot showing the fifth cycle and the third presenting the tenth cycle. It can be seen that the current increases with increasing cycle number, which is consistent with polymer growth. The peaks labeled as A1 and A2 correspond to the oxidation of the polymer from the leucoemeraldine to the emeraldine redox states and from the emeraldine to the pernigraniline states respectively. The peaks labeled as $\mathrm{C} 2$ and $\mathrm{C} 1$ correspond to the reduction of the polymer from the pernigraniline to the emeraldine and from the emeraldine to the leucomeraldine states, respectively. For comparative purposes, data recorded at aluminium in the aniline-free tosylic acid solution are shown in Fig. 4(b). For ease of comparison, these data are shown on the same scale as the data in Fig. 4(a). These plots are considerably different to those obtained in the presence of aniline. The anodic currents, which can be ascribed to the oxidation of aluminium, are low. The first cycle is shown in the insert in Fig. 4(b). During the forward scan, the current increases in magnitude with increasing anodic potential to reach a value of approximately $3 \mathrm{~mA}$ at $1.0 \mathrm{~V}$ (SCE). The current decreases in magnitude during the reverse scan. This is consistent with the formation of an anodized layer on the aluminium electrode, with the low currents during the reverse scan and subsequent cycles arising because of the passivating nature of the anodized layer. 

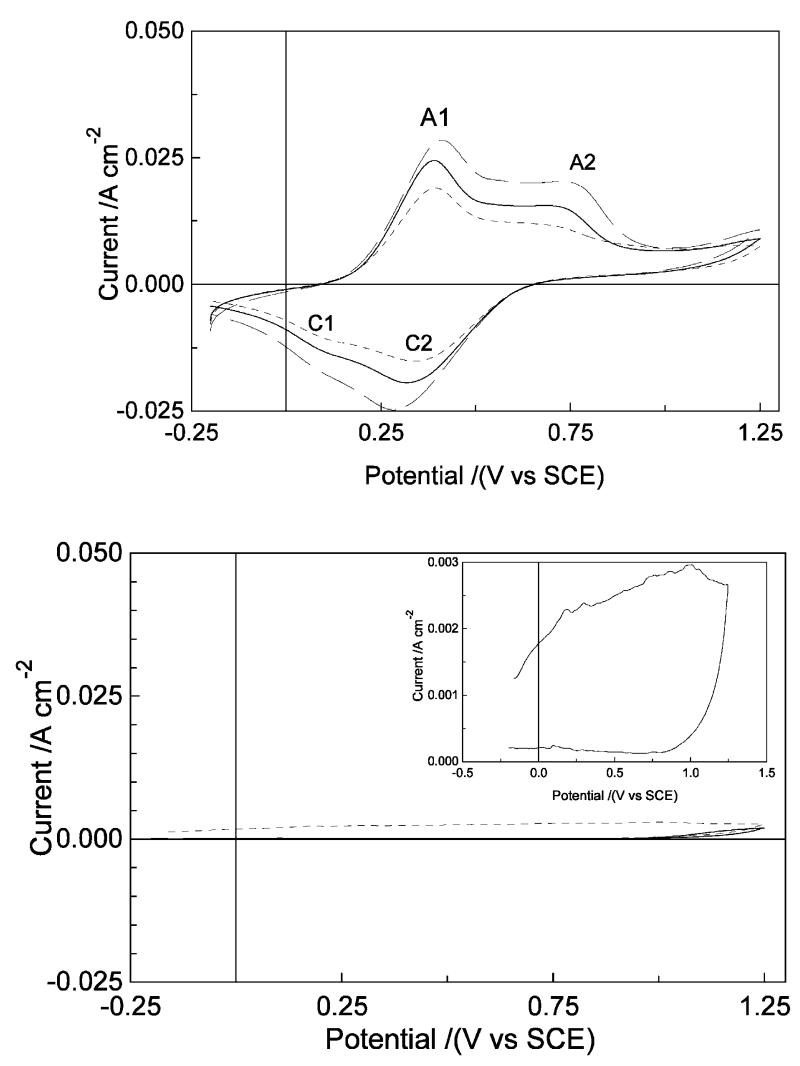

Fig. 4. (a) Cyclic voltammograms recorded for aluminium cycled at 50 $\mathrm{mV} \mathrm{s}^{-1}$ in a $0.134 \mathrm{~mol} \mathrm{dm}^{-3}$ aniline, $1.0 \mathrm{~mol} \mathrm{dm}^{-3}$ tosylic acid solution: ----, second cycle; - , fifth cycle and, - - - tenth cycle. (b) Cyclic voltammograms recorded for aluminium cycled at $50 \mathrm{mV}$ $\mathrm{s}^{-1}$ in $1.0 \mathrm{~mol} \mathrm{dm}^{-3}$ tosylic acid solution: ----, first cycle; - second cycle.

Both these procedures gave rise to the deposition of a dark green/black polymer, characteristic of polyaniline in the emeraldine/pernigraniline oxidation states, on the surface of the aluminium electrode. The outer layers of the polymer could be removed, but the underlying layers were adherent.

\subsection{Polymer characterization}

The molecular masses of these polymers were determined using gel permeation chromatography and narrow molecular weight polystyrene standards. For comparative purposes, similar measurements were performed on a polyaniline film formed from $\mathrm{H}_{2} \mathrm{SO}_{4}$ on pure platinum. This film was deposited using cyclic voltammetry, scanning the potential between the limits of -200 and $750 \mathrm{mV}$ (SCE). This procedure, using relatively low anodic potentials, ensured that the polyaniline deposit was pure and free of degradation, or decomposition, products. A typical chromatogram is shown in Fig. 5 for the polyaniline deposited at the aluminium sample. The characteristic bimodal molecular weight distribution, which has been reported previously for polyaniline, in the form of the emeraldine base $[17,18]$, is evident. The presence of the first peak, i.e. the high molecular weigh fraction, has been attributed to aggregates of polymer chains linked through hydrogen bonding, This aggregation is the result of inter-chain hydrogen bonding between the amine and imine nitrogen atoms in the polyaniline backbone $[17,18]$. These data are similar to those reported by MacDiarmid and co-workers [17] for chemically synthesized polyaniline in the emeraldine base state. Furthermore, near identical data were recorded for the polymer samples formed on platinum and aluminium. The number average molecular masses recorded for the polyaniline deposited at aluminium and platinum were $60.7 \times 10^{3}$ and $59.9 \times 10^{3} \mathrm{amu}$, respectively. The area of the high molecular weight fraction accounted for $11 \%$ of the total area for both the aluminium and platinum samples. This shows that there is very little variation between both systems, with similar hydrogen bonding and polymer chain lengths being recorded even though the electrosynthesis conditions are very different for the aluminium and platinum systems.

The acid-base properties of the polymer were also studied and it was found that a measurable change in the $\mathrm{pH}$ of the solution could be observed following immersion of the polyaniline-coated aluminium electrode in solution, indicating that the coating undergoes a dedoping process. For these measurements, the $\mathrm{pH}$ of a $6 \times 10^{-5} \mathrm{~mol} \mathrm{dm}{ }^{-3} \mathrm{NaCl}$ solution in which a polyaniline-coated aluminium or polyaniline-coated platinum electrode was immersed, were measured and recorded as a function of the immersion period. These data are presented in Fig. 6. A rapid decrease in the $\mathrm{pH}$ is seen during the early stages of immersion, typically after 5 $\mathrm{min}$, in the case of the polyaniline-Al system, whereas a

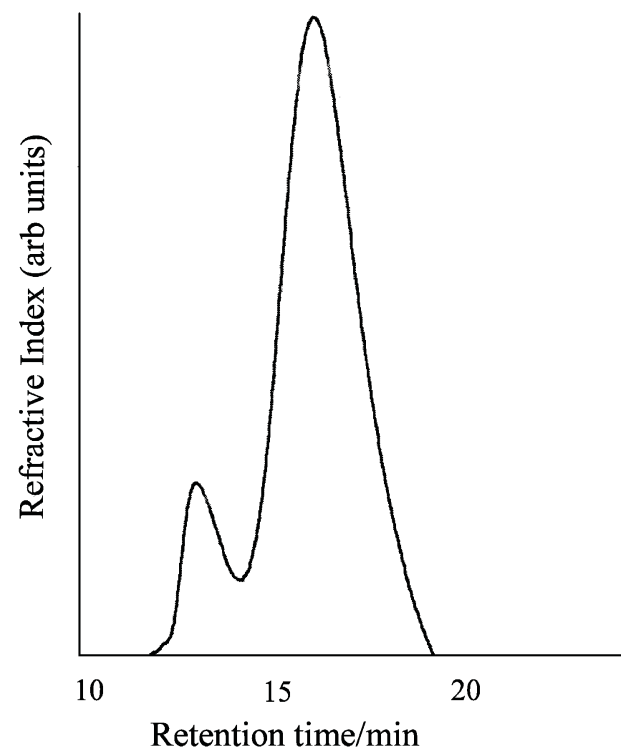

Fig. 5. A gel-permeation chromatogram recorded for polyaniline electro-synthesized at aluminium. 
more gradual reduction, typically over $40 \mathrm{~min}$, in the $\mathrm{pH}$ is evident with the polyaniline-Pt system. The steadystate $\mathrm{pH}$ values adopted by both systems are similar, indicating that the polymers formed in both cases undergo a de-doping process where $\mathrm{H}^{+}$ions are released into the solution environment. However, this also suggests that a fraction of the formed polymer resides in the emeraldine salt (ES) state, Fig. 1, which is deprotonated in the chloride electrolyte thereby causing a decrease in the $\mathrm{pH}$. The more rapid decrease in the $\mathrm{pH}$ of the polyaniline-coated aluminium electrode during the early stages of immersion may be connected with oxidation of the aluminium substrate. The ES form of the polymer is very permeable to water molecules that are readily available at the interface, the latter can oxidize the metal substrate resulting in the formation of alumina and hydrogen ions [13] (Eq. (1)).

$2 \mathrm{Al}+3 \mathrm{H}_{2} \mathrm{O} \leftrightharpoons \mathrm{Al}_{2} \mathrm{O}_{3}+6 \mathrm{H}^{+}+6 \mathrm{e}^{-}$

This reaction may also be driven by the reduction of the polymer from the ES to the leucoemeraldine salt (LS),

$3 \mathrm{ES}+6 \mathrm{H}^{+}+6 \mathrm{e} \leftrightharpoons 3 \mathrm{LS}$

The LS form can then dissociate to the LB form of the polymer, thus generating a net gain of six protons consistent with the observed decrease in $\mathrm{pH}$.

$3 \mathrm{LS} \leftrightharpoons 3 \mathrm{LB}+6 \mathrm{H}^{+}$

Following these immersion experiments the polymer became bright green in color. This transformation from dark green/black would seem to suggest that some of the polymer has undergone reduction to the leucoemeraldine state, which is known to be colorless.

In order to explore the actual state of the polyaniline film further, the UV-visible spectra of the polymers were recorded in NMP. A representative UV-visible spectrum recorded for polyaniline deposited at aluminium and then dissolved in NMP is shown in Fig. 7. The spectrum exhibits two absorption bands, one centered at $320 \mathrm{~nm}$ and the other at $626 \mathrm{~nm}$. Although there is some

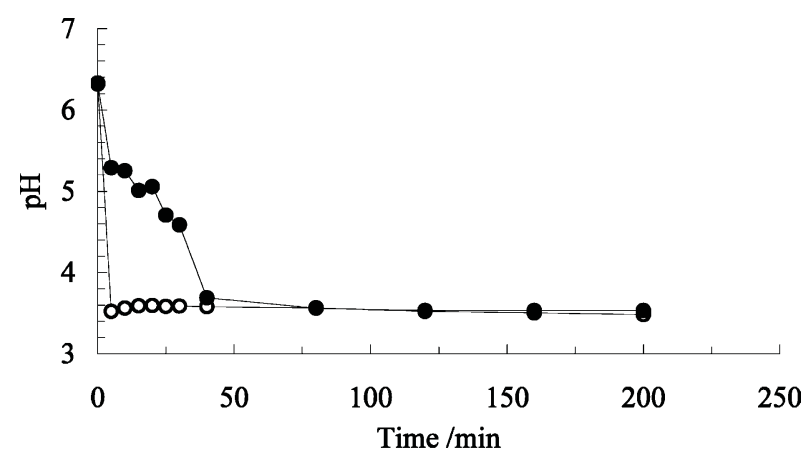

Fig. 6. $\mathrm{pH}$ as a function of time for, $\bigcirc$, polyaniline-coated aluminium and, polyaniline-coated platinum immersed in a $6.0 \times 10^{-3} \mathrm{~mol}$ $\mathrm{dm}^{-3} \mathrm{NaCl}$ solution. ambiguity in the assignment of these bands, particularly the band at $626 \mathrm{~nm}$, most researchers accept that the transition at $320 \mathrm{~nm}$ corresponds to a $\pi-\pi^{*}$ transition, while the intensity of the band at $626 \mathrm{~nm}$ depends on the oxidation state of the polymer $[19,20]$. However, the most interesting aspect of this spectrum is that it is consistent with the emeraldine base form of the polymer, exhibiting two characteristic absorption bands. In contrast, the absorption spectrum for the ES consists of four absorption bands centered at 335, 580, 615 and 870 $\mathrm{nm}$. Although wavelengths of $870 \mathrm{~nm}$ are not shown on the plot in Fig. 7, there was no evidence of any absorption band at this wavelength, confirming that the polymer dissolved in this NMP solution is predominantly the emeraldine base. This in conjunction with the $\mathrm{pH}$ data suggests that the polymer formed contains a mixture of the ES and base. Indeed, there is evidence that emeraldine adopts a structure in which metallic islands of protonated ES coexist with the unprotonated base form [21]. The fact that the salt form is not seen in the UV-visible spectrum may be associated with the very low solubility of the ES in organic solvents, making the NMP solution rich in the base form. Alternatively, the lack of an acidic environment may inhibit the polymer from adopting the salt form.

\subsection{Electrochemical and corrosion measurements}

Once formed and characterized, these polymers were subjected to a series of electrochemical tests and corrosion measurements. In Fig. 8(a and b), cyclic voltammograms recorded for polyaniline-coated aluminium and polyaniline-coated platinum in the monomerfree tosylic acid solution are shown. For the aluminium system, three plots are shown in the figure, representing voltammograms recorded at 10,50 and $70 \mathrm{mV} \mathrm{s}^{-1}$. In these measurements, the potential was cycled only to a vertex of $0.75 \mathrm{~V}$ (SCE) in order to avoid anodic degradation of the polymer. The characteristic peaks, labeled, as A1, A2, C1, and C2, evident during formation of the polymer, in Fig. 4, are less clear in this aniline-free solution. Furthermore, the scan rate appears

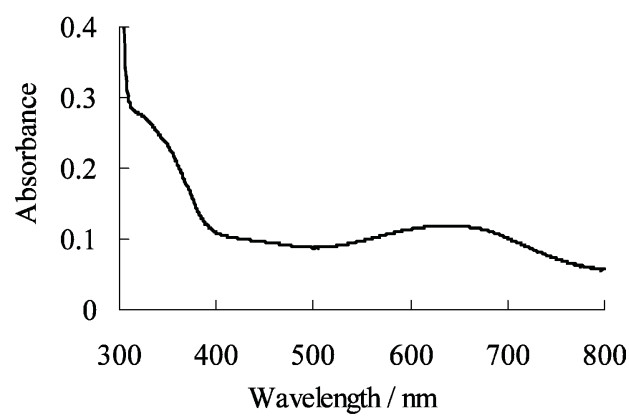

Fig. 7. UV-visible spectrum recorded for polyaniline electrosynthesized at aluminium. 

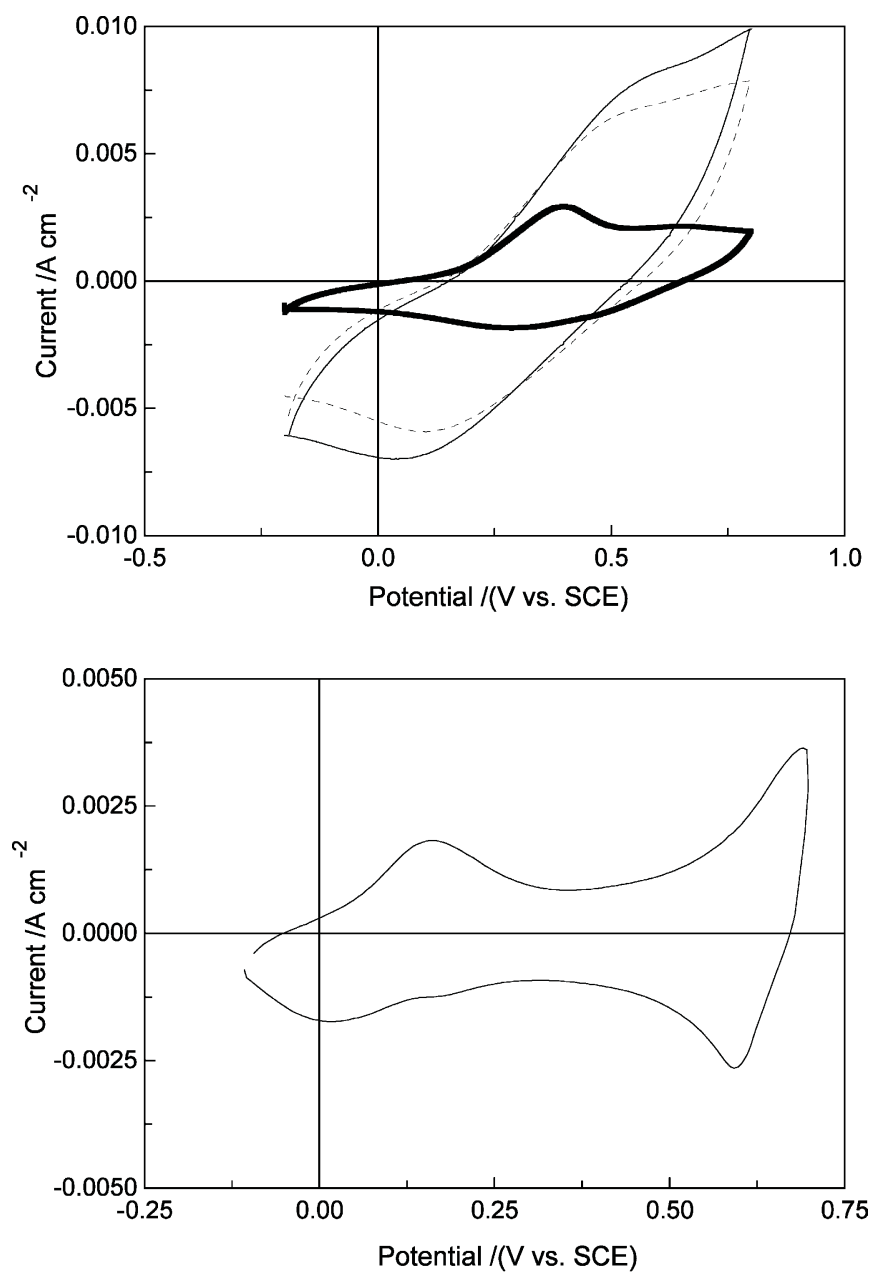

Fig. 8. (a) Cyclic voltammograms recorded for polyaniline-coated aluminium in $1.0 \mathrm{~mol} \mathrm{dm}{ }^{-3}$ tosylic acid solution recorded at: $\cdots$, 10 ; --- 50 and, -, $70 \mathrm{mV} \mathrm{s}^{-1}$. (b) Cyclic voltammograms recorded for polyaniline-coated platinum in $1.0 \mathrm{~mol} \mathrm{dm}{ }^{-3}$ tosylic acid solution recorded at $50 \mathrm{mV} \mathrm{s}^{-1}$.

to have a significant effect on the electrochemical response. An oxidation peak centered at approximately $325 \mathrm{mV}$ (SCE), which probably represents oxidation of the polymer from the leucoemeraldine to the emeraldine state, is seen clearly only at a low scan rate of $10 \mathrm{mV}$ $\mathrm{s}^{-1}$, while the second oxidation peak is not evident at any of the scan rates. Even on polarizing the polyaniline-aluminium electrode to higher potentials, this second oxidation peak was not observed. But, more significantly, the polymers appear to loss their electroactivity at the higher scan rates.

These data are very similar to those reported for polyaniline deposited at inert electrodes, cycled in solutions with $\mathrm{pH}$ values greater than 4.0 [22,23]. In some of these studies, it has been suggested that deprotonation of the ES, in such high $\mathrm{pH}$ media, is responsible for the observed loss of electroactivity. However, this is not the case for this aluminium system, as the two oxidation peaks, and corresponding reduc- tion peaks, characteristic of polyaniline, are well resolved for the platinum system, Fig. 8(b). Furthermore, this indicates that the lack of electroactivity observed for the aluminium-polyaniline system is not due to the size of the medium-sized tosylate anion and its restricted diffusion through the polymer. Therefore, the lack of defined electroactivity is more likely due to the insulating properties of the underlying aluminium oxide layer, or to degradation, or overoxidation, of the polyaniline film. The degradation of polyaniline can be identified easily in cyclic voltammetry experiments by the presence of a 'middle peak' lying between the leucoemeraldine and emeraldine and the emeraldine and pernigraniline couples. This 'middle' peak has been taken as evidence for the build-up of degradation products, for film overoxidation [24], to residual oligomers trapped within the film [25] and for different polymer backbone structures arising from ortho coupling [26]. In Figs. 4 and 8 , this middle peak is not observed, indicating that the high anodic potentials employed in the electrosynthesis reactions do not lead to appreciable degradation of the polymer. Therefore, it appears that the loss in electroactivity is more likely connected to the oxidized aluminium substrate and the insulating properties of this oxide.

In Fig. 9(a), electrochemical impedance data are presented for the polyaniline-coated aluminium electrode and polyaniline-coated platinum immersed under

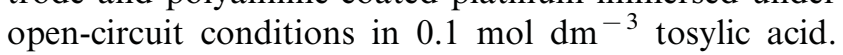
The data presented for the aluminium system were recorded following 2.6 and $30 \mathrm{~h}$ immersion in the tosylic acid solution, while the data presented for the platinum system were recorded following $1 \mathrm{~h}$ immersion, although near identical data were obtained for the polyaniline- $\mathrm{Pt}$ system as a function of immersion time. These data, presented as Nyquist plots, show that the impedance response is very different for the polyaniline deposited on aluminium and on platinum. The data shown for the platinum system are typical of conducting polymers and can be fit to the equivalent circuit depicted in Fig. 9(b). In this circuit a serial combination of a resistor and a constant phase element, Q1, is used to represent the polyaniline-Pt system. The resistance term $\mathrm{R} 1$, which corresponds to the high frequency limit of the impedance, has been analyzed by several authors [27-29] and has been attributed to the solution resistance and the ionic and electronic resistance of the polymer. The constant phase element, which is represented by Q1, had an exponent $n$ value of the order of 0.91 , which equates to a near ideal capacitor. This capacitance behavior is seen in the low frequency region and is a very characteristic feature of oxidized polyaniline. It has been explained in terms of the intrinsic redox charging within the polymer film $[27,28]$, although other explanations have been put forward. However, the data recorded for the polyaniline-Al system are very differ- 


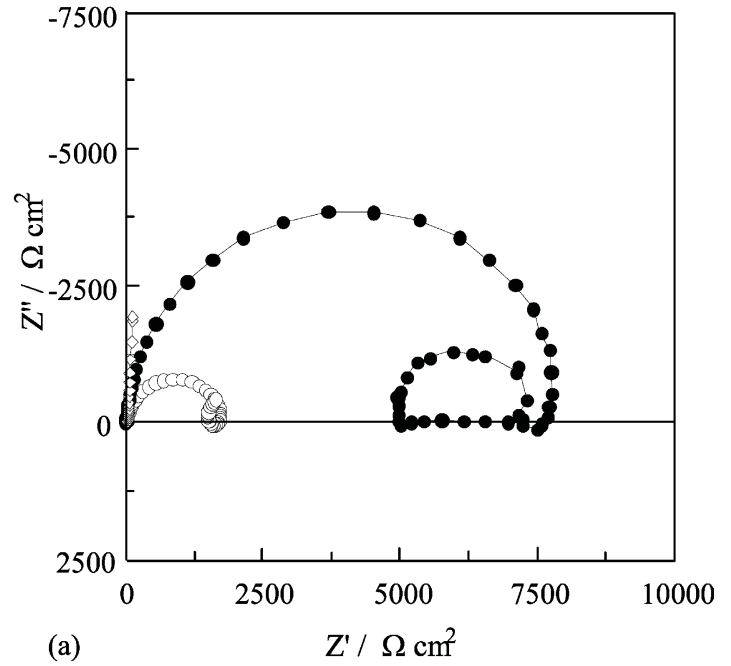

(b)

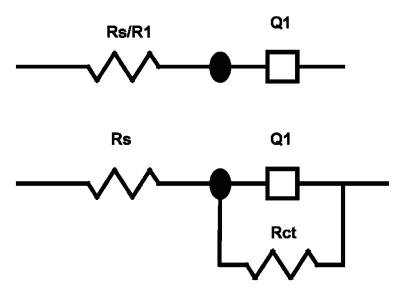

(c)

Fig. 9. (a) Impedance data recorded for polyaniline-coated aluminium in a $1.0 \mathrm{~mol} \mathrm{dm}^{-3}$ tosylic acid solution following $\bigcirc 30 \mathrm{~min}$ and $\bullet 30$ $\mathrm{h}$ and $\diamond$ polyaniline-coated platinum. (b) Equivalent circuit for platinum system. (c) Equivalent circuit for aluminium system.

ent and, as shown in Fig. 9(a), change on immersion. These data shown for the polyaniline-Al system were recorded from $65 \mathrm{kHz}$ to $0.05 \mathrm{~Hz}$ and consist of a single arc that can be generated by a simple Randles cell, comprising a solution resistance, $R_{\mathrm{s}}$, a double layer capacitance, $Q_{1}$, and a charge transfer resistance, $R_{\mathrm{ct}}$, Fig. 8(c). Although not shown here, lower frequency data give rise to an inductive loop that may be associated with the formation of a salt film. The charge transfer resistance computed for the polyaniline-Al system following $2 \mathrm{~h}$ of immersion was $1730 \Omega \mathrm{cm}^{2}$, but following $30 \mathrm{~h}$ of immersion, the charge-transfer resistance had increased to $8030 \Omega \mathrm{cm}^{2}$. Likewise, the double layer capacitance decreased from $3.7 \times 10^{-5}$ to $9.1 \times 10^{-6} \mathrm{~F} \mathrm{~cm}^{-2}$ during the same period of immersion. Initially, the impedance spectrum is dominated by the conducting properties of the polyaniline and to a lesser extent the aluminium substrate. But, even at this early period, there is a contribution from the aluminium substrate as can be seen from a comparison of these data with those shown for the polyaniline-Pt system. On further immersion, oxidation of the aluminium substrate occurs resulting in an increasing thicker oxide layer at the metal-conducting polymer interface and reduction of the polyaniline layer to the less conducting leucoemeraldine form, as evidenced by an increase in the charge transfer resistance and a decrease in the capacitance. Indeed, clear evidence for the aluminium oxidation reaction could be obtained by removing the polymer coating. The substrate appeared white in coloration, which is consistent with the oxidation of aluminium and the generation of a thick $\mathrm{Al}_{2} \mathrm{O}_{3}$ oxide (Eq. (1)).

In order to probe the corrosion protection properties of the deposited polyaniline, anodic polarization measurements and local surface potential measurements, recorded using a scanning vibrating electrode technique, were performed. Representative polarization plots recorded for uncoated aluminium and polyaniline coated aluminium in a highly aggressive $0.5 \mathrm{~mol} \mathrm{dm}{ }^{-3} \mathrm{NaCl}$, pH 5.85 electrolyte, are shown in Fig. 10. The plot depicted for the uncoated aluminium electrode is typical of the cathodic and anodic polarization behavior of pure aluminium; the corrosion potential lies at $-1.24 \mathrm{~V}$ (SCE), at higher potentials a passive region, with an average current of $3 \mu \mathrm{A} \mathrm{cm}{ }^{-2}$ is obtained, and breakdown and pitting of the electrode occurs at $-750 \mathrm{mV}$ (SCE). The polarization behavior of the polyanilinecoated aluminium electrode is very different. Cathodic reactions dominate the electrochemical response in the potential region between -1600 and $-715 \mathrm{mV}$ (SCE). Although Kinlen et al. [30] and Tallman et al. [13] have found that polyaniline can mediate oxygen reduction at the polymer electrolyte interface, this oxygen reduction reaction only accounts for a small fraction of this current. This was confirmed by recording the cathodic polarization behavior of polyaniline-coated aluminium in oxygenated and oxygen-free solutions. Although the current decreased by a factor of two in the oxygen-free solution, the cathodic current recorded for the polyaniline-coated aluminium electrode in the oxygen-free solution was a factor of ten greater than that observed for bare aluminium. This tends to suggest that under these cathodic polarization conditions that hydrogen ion reduction and reduction of the polymer from the emeraldine state to the leucoemeraldine state account

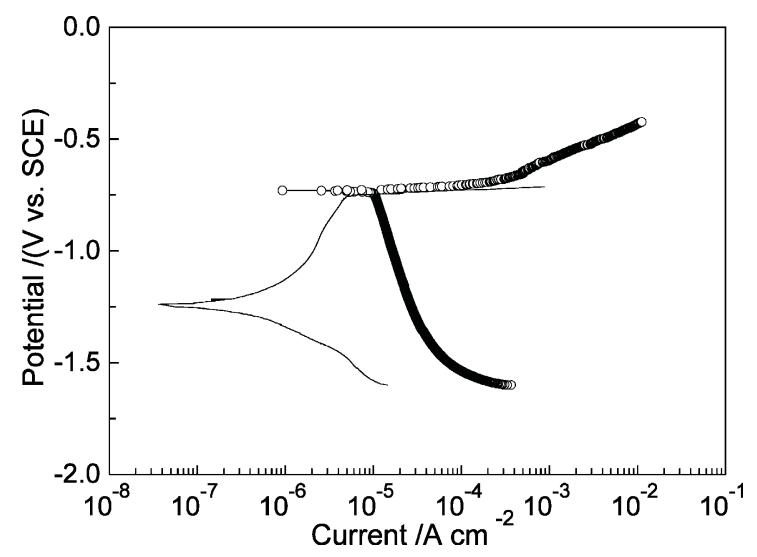

Fig. 10. Potentiodynamic polarization plots recorded in $0.5 \mathrm{~mol} \mathrm{dm}^{-3}$ $\mathrm{NaCl}$ for, - , pure aluminium and, $\bigcirc$, polyaniline-coated aluminium. 
for some of the measured current. On polarizing further in the anodic direction, the mixed potential and the anodic branch are observed. The mixed potential lies at $-715 \mathrm{mV}$ (SCE) and this is then followed by a sharp increase in the anodic current up to an approximate value of $3 \times 10^{-4} \mathrm{~A} \mathrm{~cm}^{-2}$ at which point the rate of increase in the anodic current is reduced giving an inflection in the plot. This inflection in the current potential data seems to be associated with diffusion of the corrosion products away from the electrode surface. The polymer, although enabling diffusion of chloride anions to the aluminium interface, seems to limit the diffusion of the aluminium chloride corrosion products, possibly because of size constraints, from the interface into the bulk solution.

There is little evidence to be gained from these data to support a corrosion protection mechanism for the polyaniline coating. There is an increase in the breakdown potential of approximately $35 \mathrm{mV}$, but it is seen also that much higher rates of reduction and much higher mixed currents are accommodated at the polyaniline-Al composite. This can be seen more clearly from the data presented in Fig. 11 where a plot showing this mixed current as a function of the chloride concentration for polarization of aluminium and the polyaniline aluminium composite are presented. The mixed current shown for the uncoated aluminium electrode is equivalent to the corrosion current marking high rates of corrosion with increasing chloride concentration, while the origin of the mixed currents shown for the polyaniline-aluminium composite are less clear. These currents appear to be dominated by the high rates of reduction and the ability of the polymeraluminium composite to catalyze reduction reactions (Fig. 10).

In order to study the galvanic interaction between the polymer and the aluminium substrate, the polymer was removed from part of the aluminium substrate and the surface potential across the coated and uncoated areas was mapped as a function of the potential applied to the electrode. Data from these surface potential measurements, obtained using a scanning vibrating probe technique, are shown in Fig. 12(a-c), as a function of

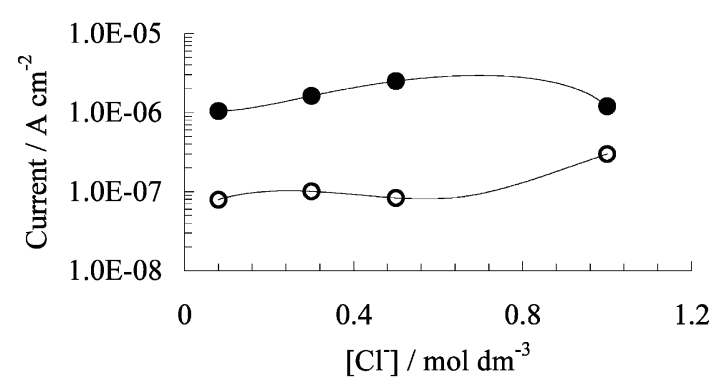

Fig. 11. Mixed current plotted as a function of chloride concentration for, $\bigcirc$, polyaniline-coated aluminium and, $\bigcirc$, pure aluminium in a neutral $\mathrm{NaCl}$ solution. the potential applied to the composite electrode. The uncoated area is marked by the circular area extending from 1580 to $0 \mu \mathrm{m}$ ( $y$-axis) and between 2800 and 4200 $\mu \mathrm{m}$ ( $x$-axis) on the displacement scale. The corresponding bulk current, measured at each of the potentials, is shown in Fig. 12(d). For these measurements a dilute

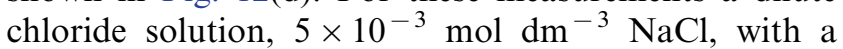
relatively low conductivity was employed. This in turn means that much higher potentials are needed to nucleate corrosion on the aluminium electrode. The image presented in Fig. 12(a) was recorded at an applied potential of $-800 \mathrm{mV}$ (SCE) which corresponds to a potential at which the polyaniline-coated aluminium electrodes are stable and the bulk current recorded is a reduction current, Fig. 12(d). There is very little variation, less than $50 \mu \mathrm{V}$, in the surface potential in this case. It is also interesting to note that there is no variation between the coated and uncoated surfaces, showing that both surfaces are polarized to a common mixed potential. Similar data were recorded at $-600 \mathrm{mV}$ (SCE) showing that the polymer offers some corrosion protection to the underlying substrate under these conditions.

However, on increasing the potential to a value of $0.4 \mathrm{~V}$ (SCE), Fig. 12(b), breakdown of the electrode occurs. A bulk anodic current close to $0.1 \mathrm{~mA} \mathrm{~cm}^{-2}$ is recorded. Even though this high anodic current is measured there is no evidence of any variation between the surface potential of the coated and the adjacent uncoated parts of the electrode. Instead, it appears that the uncoated surface and the regions adjacent to this site dissolve at a similar rate and that the polymer offers little in terms of any corrosion protection properties under these higher polarization conditions. However, areas accommodating a reduction reaction can be seen in the lower right hand corner $(7000$ and $-1050 \mu \mathrm{m}$ on the displacement scale) and in the lower center section of the image ( 2800 and $2630 \mu \mathrm{m}$ on the displacement scale). On polarizing the composite to a higher potential, although higher bulk anodic currents are measured, the area accommodated by these reduction reactions appears to increase. This is seen in Fig. 11(c), where the image was recorded at $-0.2 \mathrm{~V}$ (SCE). In this case, the difference between the surface potential measured over the cathodic centers and that on the active surface was of the order of $630 \mu \mathrm{V}$. It is interesting to note that the cathodic event initially centered at 2800 and $-2630 \mu \mathrm{m}$ on the displacement scale consumes the surface at a much greater rate than the neighboring site. Although the extent of corrosion is much greater under these conditions, the polymer appeared to remain intact.

These data are in agreement with the currentpotential behavior seen in Fig. 10, where the anodic currents measured at potentials in the region of -500 $\mathrm{mV}$ (SCE) in the $0.5 \mathrm{~mol} \mathrm{dm}{ }^{-3} \mathrm{NaCl}$ solution appear to be limited by a diffusion process. This seems to be 


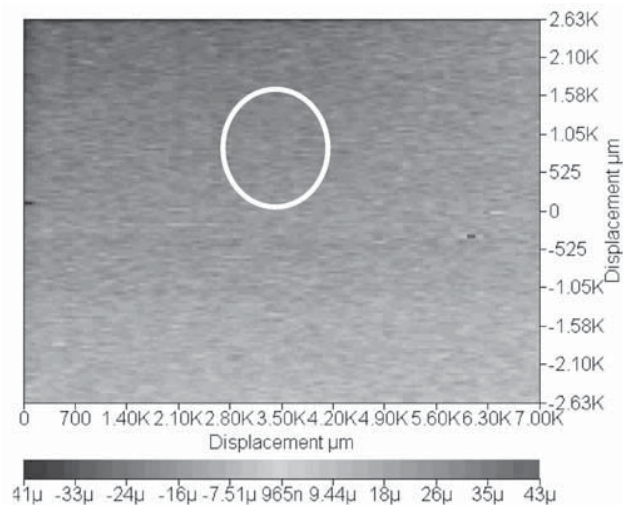

(a)
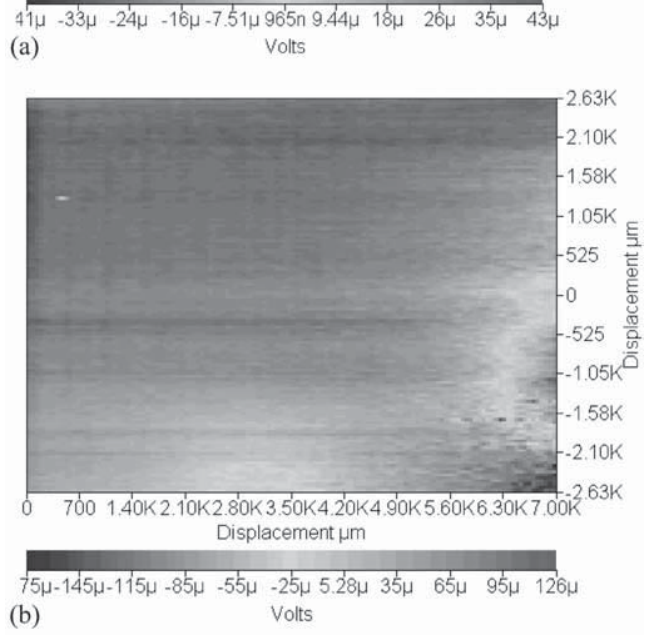
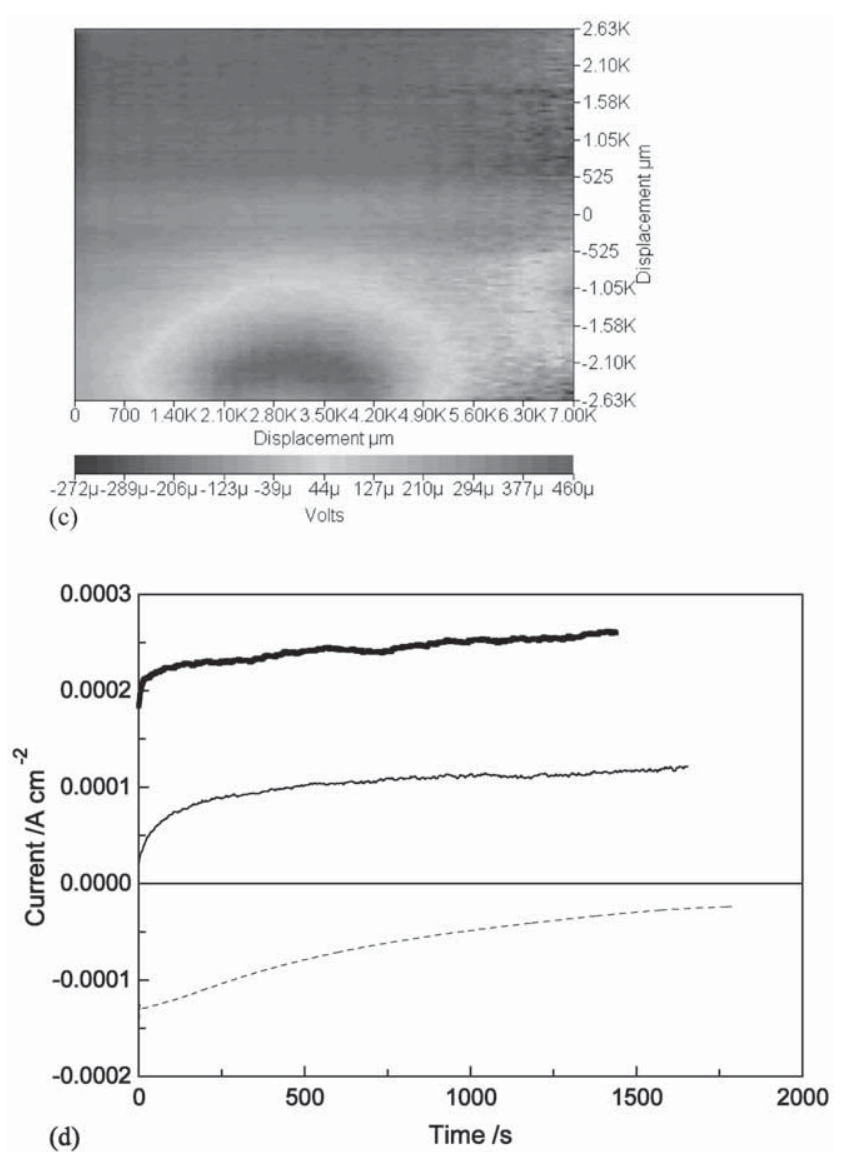

(d)

Fig. 12. Scanning vibrating probe image recorded for polyaniline-coated aluminium electrode polarized in a $6 \times 10^{-3} \mathrm{~mol} \mathrm{dm}^{-3} \mathrm{NaCl}$ solution at (a) $-0.8 \mathrm{~V}$ (SCE), (b) $-0.4 \mathrm{~V}$ (SCE), (c) $-0.2 \mathrm{~V}$ (SCE) and (d) bulk current measured at: ----, $-0.8 \mathrm{~V}$ (SCE); - , - $0.4 \mathrm{~V}$ (SCE) and, $\cdots \cdots,-0.2 \mathrm{~V}$ (SCE).

connected with dissolution of the underlying aluminium substrate and diffusion of these corrosion products through the polymer coating and into the bulk solution. Dissolution of the underlying aluminium substrate will lead to local detachment of the polymer from the substrate. The presence of the cathodic sites, Fig. 12(b and c), is consistent with reduction of the detached polymer form the emeraldine to the leucoemeraldine states, with this reduction reaction being catalyzed by the aluminium oxidation reaction. The expanding area accommodated by the reduced polymer on increasing the potential applied to the composite is consistent with the much higher dissolution rates and higher rates of polymer detachment at the higher potentials.

\section{Discussion}

It can be seen from the results presented that polyaniline coatings can be deposited onto pure aluminium in the presence of tosylic acid using either a constant potential or a potential cycling regime. As evident from
Fig. 3, the rate of the electro-polymerization reaction depends on the aniline concentration and the applied potential. This is consistent with the first step in the reaction being the generation of radical cations, which in turn is facilitated by higher anodic potentials. However, there is a secondary electrochemical reaction; the oxidation of the aluminium substrate.

It is commonly accepted that oxidation of aluminium occurs in acidic media to generate the well-known duplex structure, with an inner barrier layer and an outer porous layer [31]. Husler and Beck [6,7] have shown that these pores play an important role in the electropolymerization of pyrrole at an aluminium substrate. Although the pores are small with diameters between 1.5 and $3 \mathrm{~nm}$, they provide an initial current path for the electrochemical reaction, giving rise to the nucleation of polypyrrole within the pores. With continued polymerization, the polymer extends over the entire surface. It is likely that similar events occur during the electropolymerization of aniline at aluminium, with the initial nucleated polyaniline residing within the pores. However, as Husler and Beck [6,7] have pointed 
out, the geometrical current density within these pores will be amplified considerably and this will lead to an initial overoxidized or degraded polyaniline, which in turn is likely to exhibit a low electronic conductivity. Nevertheless, polyaniline is further nucleated over these initial deposits to generate a conducting polymer coating.

Despite the very different electrosynthetic conditions that exist during deposition of polyaniline at aluminium and at platinum, the conducting polymers exhibit surprisingly similar characteristics when removed from the electrode surface. Furthermore, the distribution of molecular masses, recorded for the aluminium and platinum system were very similar to the measurements made by MacDiarmid and co-workers [17,18] on chemically synthesized polyaniline. In both studies a bimodal distribution, Fig. 5, was obtained, with similar fractions of the high molecular weight chains. These data are interesting on another front. It is known that with degradation of polyaniline, chains with lower molecular mass are formed [32]. However, the number average molecular mass calculated for the aluminium system was in fact higher than that calculated with the platinum system, indicating no significant decrease in the molecular weights of the chains formed on the aluminium electrodes. In addition, similar UV-visible spectra were recorded for the polyaniline deposited at aluminium and platinum. Furthermore, these spectra showed that much of the polyaniline dissolved in the NMP solution resided in the emeraldine base form. However, as already discussed, the polymer probably adopts a structure in which metallic islands of protonated ES coexist with the unprotonated base form [21]. Indeed, the $\mathrm{pH}$ data presented in Fig. 6 support such a conclusion.

Although oxidation of the aluminium substrate occurs during growth of the polymer, it also appears that further oxidation of the aluminium occurs coupled with reduction of the polymer on immersion of the composite in solution, giving rise to a galvanic interaction between the polymer and substrate. This is seen in the electrochemical data presented in Figs. 6, 8 and 9. In particular, the time dependent electrochemical impedance data measured for the aluminium-polyaniline system is consistent with reduction of the polymer and oxidation of the aluminium substrate. The charge transfer resistance is found to increase and the capacitance to decrease with increasing immersion, indicating a loss of conductivity of the polyaniline film through reduction of the film and the corresponding oxidation of the aluminium substrate. Indeed, these data recorded under extended immersion times were very similar to those recorded for uncoated aluminium, showing that the impedance response is dominated by the properties of the oxidized aluminium substrate.
Other authors have observed a similar galvanic interaction $[13,33]$. For example, Cogan et al. [33] have concluded from their measurements of galvanic couples between polyaniline doped with camphorsulfonic acid and AA 2024-T3 that the alloy dominates the mixed potential response and polarizes the polyaniline to values near the potential of the freely corroding alloy, indicating that the conducting polyaniline coatings are reduced to the nonconductive form. They have also suggested that any corrosion protection of the alloy by the polyaniline is due to the barrier properties of the reduced polymer.

As evident from Figs. 10-12, there is little evidence of any significant corrosion protection of the aluminium substrate by the electrodeposited polyaniline coatings. There was a slight increase in the breakdown potential, approximately $35 \mathrm{mV}$, for the polyaniline-coated electrodes. Saidman and Bessone [9] have shown that the pitting potential recorded for polypyrrole-coated aluminium depends on the electrosynthesis conditions, with lower pitting potentials being recorded for coatings formed at higher potentials due to degradation of the polypyrrole. However, for this polyaniline system, the breakdown potentials appeared to be independent of the electrosynthesis conditions. Films formed at lower potentials of $750-800 \mathrm{mV}$, which are likely to be completely free of any degradation products, had in fact slightly lower breakdown potentials. However, this is probably connected to the fact that these films were 'thinner' than those formed at $1.25 \mathrm{~V}$ (SCE).

Since breakdown of the aluminium substrate occurs in the potential region of $-700 \mathrm{mV}$ (SCE), then the polyaniline film is maintained in the reduced leucoemeraldine state. Under these conditions the polymer will act only as a barrier coating, in agreement with the conclusions reached by Cogan et al. [33]. It is likely that in the presence of the chloride anions, that some of the tosylate anions are exchanged with the chloride anions and these migrate to the aluminium substrate causing dissolution of the substrate.

It is also seen in Fig. 11, that much higher mixed currents are recorded for the polyaniline-coated electrodes. The mixed currents shown for the aluminium electrode are equivalent to the corrosion currents. However, the values shown for the polyaniline-coated aluminium are more difficult to analyze, as they are likely to be governed by a number of contributions. These experiments were performed following formation of the polymer and hence the polymer will exist as the ES/base. Upon application of a cathodic potential of $-1.6 \mathrm{~V}$ (SCE) the polymer will undergo a reduction reaction to the leucoemeraldine form, the polymer can also catalyze the oxygen reduction reaction [30] and reduction of hydrogen may also occur at the polymer or at the aluminium substrate. However, these additional reduction reactions will drive the aluminium oxidation 
reaction. Although this may be beneficial, it is seen from the data presented in Fig. 12 that dissolution of this oxidized aluminium substrate can occur and that this attack propagates underneath the polymer leading to detachment of the polymer and reduction of the detached polymer.

\section{Conclusions}

Polyaniline films were electrodeposited at pure aluminium from a tosylic acid solution. These polymer films exhibited similar characteristics as pure polyaniline electrosynthesized at an inert platinum electrode, when removed from their respective substrates. Both polymers had similar molecular weights and similar UV-visible absorption spectra. However, their electrochemical properties were somewhat different indicating that the substrate plays an important role in determining these properties. A galvanic interaction between the polymer and the aluminium was observed giving rise to oxidation of the aluminium substrate and reduction of the polymer. Although the aluminium substrate was oxidized there was little evidence of any corrosion protection by the polyaniline coatings. On polarizing the electrode, attack at the aluminium substrate occurred underneath the polymer, indicating that chloride anions diffuse across the polymer to react at the underlying substrate.

\section{Acknowledgements}

The authors gratefully acknowledge the support of this work by Enterprise Ireland, under the Basic Science Research Grants Award, Project Code SC/99/106.

\section{References}

[1] D.T. Hoa, T.N. Suresh Kumar, R.S. Srinisava, R. Iai, N.S. Punekar, Anal. Chem. 64 (1992) 2465.

[2] J. Heinze, Synth. Met. 41-43 (1995) 2805.
[3] M.C. Bernard, A. Hugot-Le Goff, S. Joiret, N.N. Dinh, N.N. Toan, J. Electrochem. Soc. 146 (1999) 995.

[4] D.E. Tallman, G. Spinks, A. Dominis, G.G. Wallace, J. Solid State Electr. 6 (2002) 73.

[5] J.O. Iroh, W.C. Su, Electrochim. Acta 46 (2000) 15.

[6] P. Husler, F. Beck, J. Appl. Electrochem. 20 (1990) 596.

[7] F. Beck, P. Husler, J. Electroanal. Chem. 280 (1990) 159.

[8] D.E. Tallman, C. Vang, G.G. Wallace, G.P. Bierwagen, J. Electrochem. Soc. 149 (2002) C173.

[9] S.B. Saidman, J.B. Bessone, J. Electroanal. Chem. 51 (2002) 87.

[10] K. Naoi, M. Takeda, H. Kanno, M. Sakakura, A. Shimada, Electrochim. Acta 45 (2000) 3413.

[11] A.J. Epstein, J.A.O. Smallfield, H. Guan, M. Fahlman, Synth. Met. 102 (1999) 1374.

[12] R. Racicot, R. Brown, S.C. Yang, Synth. Met. 85 (1997) 1263.

[13] D.E. Tallman, Y. Pae, G.P. Bierwagen, Corrosion 56 (2000) 401.

[14] K. Shah, Y. Zhu, G.S. Akundy, J.O. Iroh, O. Popoola, Key Eng. Mat. 197 (2001) 111.

[15] A. Eftekhari, Synth. Met. 125 (2001) 295.

[16] A. Eftekhari, Sensors Actuators B 80 (2001) 285.

[17] W. Zheng, M. Angelopoulos, A.J. Epstein, A.G. MacDiarmid, Macromolecules 30 (1997) 2953.

[18] W. Zheng, M. Angelopoulos, A.J. Epstein, A.G. MacDiarmid, Macromolecules 30 (1997) 7634.

[19] P. Swapna Rao, J. Anand, S. Palaniappan, N. Sathyanarayana, Eur. Polym. J. 36 (2000) 915.

[20] J.E. Albuquerque, L.H.C. Matttoso, D.T. Balogh, R.M. Faria, J.G. Masters, A.G. MacDaiarmid, Synth. Met. 113 (2000) 19.

[21] A.J. Epstein, A.G. MacDiarmid, Mol. Cryst. Liq. Cryst. 160 (1988) 165.

[22] T. Hirai, S. Kuwabata, H. Yoneyama, J. Chem. Soc., Faraday Trans. I 85 (1989) 969.

[23] L. Nyholm, L.M. Peter, J. Chem. Soc., Faraday Trans. 90 (1994) 149

[24] E.M. Genies, M. Lapkowski, J. Electroanal. Chem. 249 (1988) 97.

[25] S. Catarin, L. Doubova, G. Mengoli, C. Zotti, Electrochim. Acta 33 (1988) 1077.

[26] F.A. Viva, E.M. Andrade, F.V. Molina, M.I. Florit, J. Electroanal. Chem. 417 (1999) 180.

[27] E. Musiani, Electrochim. Acta 35 (1990) 1665.

[28] R.P. Buck, M.B. Madaras, R. Mackel, J. Electroanal. Chem. 362 (1993) 33.

[29] E.S. Matveeva, M.J. Gonzalez-Tejera, J. Electrochem. Soc. 147 (2000) 1213

[30] P.J. Kinlen, V. Menton, Y. Ding, J. Electrochem. Soc. 146 (1999) 3690.

[31] F. Mansfeld, C. Chen, C.B. Breslin, D. Hull, J. Electrochem. Soc. 145 (8) (1998) 2792

[32] Y. Fong, J.B. Schlenoff, Polymer 36 (1995) 639.

[33] S. Cogan, M. Gilbert, G. Holleck, J. Ehrlich, M. Jillson, J. Electrochem. Soc. 147 (2000) 2143. 